\title{
EDITORIAL
}

\section{Physical influences on stem cells}

\author{
Gordana Vunjak-Novakovic
}

We invited some of the leaders of the burgeoning field of physical regulation of stem cells to critically review biophysical regulation of stem cell fate and function in the context of tissue development and regeneration. We expect that this set of 13 excellent reviews published in several issues of the Stem Cell Research \& Therapy will be of great interest for unlocking the full regenerative potential of stem cells.

Stem cells respond to the entire context of their environment, and physical signals play major roles throughout development and adult life. Whereas the regulatory roles of morphogens have been extensively studied, physical signals are much less defined while being equally important for most of our tissues. For example, the human heart starts to beat and to pump blood through nascent blood vessels only 3 weeks into gestation. Our muscles and joints develop under rather dynamic mechanical conditions, and their well-being throughout development and adult life critically depends on physical regulatory signals.

Bioengineered environments designed to implement tissue-specific transport and physical signaling are becoming increasingly important for studying stem cells, tissue development and regeneration, and modeling disease under physiologically meaningful conditions [1-4]. More than ever before, the work at the interfaces of biology, engineering, and medical sciences is critical for advancing stem cell research and translation into new and effective clinical modalities. The path forward will almost certainly require a more profound understanding of biophysical regulation of tissues in the body, by molecular and physical signals acting in concert, with synergistic and competing effects. The complexity of cell regulation is further increased by the dynamic changes of regulatory signals. It is becoming evident that the molecular signals and physical forces combine in space and time to control cell function [2,3]. Examples of biophysical regulating factors include shear flow, compression, stretch, or electrical signals. Matrix stiffness is another

Correspondence: gv2131@columbia.edu

Department of Biomedical Engineering, Columbia University, 622 West 168th Street, VC12-234, New York, NY 10032, USA determining factor of cell fate and function [1,4]. Physical signals are also important in tissue pathologies, such as fibrous scar formation causing the stiffening of the heart muscle following myocardial infarction, which creates a mechanical environment unsuitable for normal function of therapeutic cells $[1,2]$.

In the first issues of this series, published in September 2012, two articles showcased fundamental research of physical regulation of cell function and tissue assembly and provided insightful analysis of biophysical phenomena at the cellular and tissue levels. Yim and Sheetz [5] analyzed the force-dependent cell signaling and the role of mechanobiology in stem cell differentiation. Manivannan and Nelson [6] focused on dynamic regulation of branched tissue assembly by comparing and contrasting two tubular structures: tracheal development in Drosophila and mammary development in mouse.

In the next issue, we featured two reviews that bring us back to mechanobiology at the cellular level. Conway and Schaffer [7] introduced the roles of biophysical cues in stem cells and discussed biophysical regulation of stem cells within the stem cell niche. Fridley, Kinney, and McDevitt [8] presented a review of hydrodynamic modulation of pluripotent stem cells, addressing a number of important questions - from basic biological responses to hydrodynamic shear to scaling cell expansion in dynamic culture systems. In a later issue, Wan, Ronaldson, Guirguis, and Vunjak-Novakovic [9] discussed the establishment of cell chirality (left-right asymmetry) in response to gradients and boundaries, a phenomenon critical for development and disease. We believe that these reviews will generate new ideas for utilization of physical signals in modulating stem cells and their differentiated derivatives.

Next, three exciting reviews covered physical regulation of human stem cell differentiation and assembly into three physically active tissues: vasculature, bone, and heart muscle. Serbo and Gerecht [10] discussed the design and utilization of biodegradable scaffolds for vascular tissue engineering. Hung, Hutton, and Grayson [11] examined mechanical control of bone development by coordinated regulation of cells by hydrodynamic shear, stiffness, and 
roughness of the scaffold. Thavandiran, Nunes, Xiao, and Radisic [12] analyzed the topological and electrical control of cardiac differentiation and assembly of cardiac tissues. Together, these three reviews provide new insights into the physical signaling involved in the assembly of native and engineered tissues.

Four reviews offer critical insights into the biophysical regulation of osteogenic, chondrogenic and adipogenic differentiation of stem cells. de Peppo and Marolt [13] focused on biophysical regulation of osteogenesis and maturation of mesenchymal tissue progenitors derived from human pluripotent stem cells. Chen and Jacobs [14] reviewed the roles of mechanical stimuli, mechanosensors, and regulatory pathways in mechanically induced osteogenesis of stem cells. O'Conor, Case, and Guilak [15] summarized recent findings on how chondrogenesis can be enhanced through mechanical stimulation, toward biomechanically driven tissue development and maturation of stem cell-based cartilage replacements. Schiller, Schiele, Sims, Lee and Kuo discussed the limitations of current in vitro systems to replicate adipogenesis, and described the regulatory roles of low oxygen and cytoskeletal tension [16]. Finally, Zimmermann [17] reviewed the role of mechanical loading in heart development and function and discussed the utilization of physiological loading regimens in myocardial tissue engineering.

Stem Cell Research $\mathcal{E}$ Therapy is a great forum for reporting and discussing the research and applications of stem cells. We sincerely hope that this series of reviews on physical influences on stem cells will inspire some new and interesting research and initiate further discussion. We would like very much to encourage our readers to contribute their thoughts and comments on this important aspect of stem cell research.

Note: This article is part of a thematic series on Physical influences on stem cells edited by Gordana Vunjak-Novakovic. Other articles in the series can be found online at http://stemcellres.com/series/physical.

\section{Competing interests}

The author declares that she has no competing interests.

Published: 24 Dec 2013

\section{References}

1. Engler AJ, Sen S, Sweeney HL, Discher DE: Matrix elasticity directs stem cell lineage specification. Cell 2006, 126:677-689.

2. Discher DE, Mooney DJ, Zandstra PW: Growth factors, matrices, and forces combine and control stem cells. Science 2009, 324:1673-1677.

3. Burdick JA, Vunjak-Novakovic G: Engineered microenvironments for controlled stem cell differentiation. Tissue Eng Part A 2009, 15:205-219.

4. Vunjak-Novakovic G, Scadden DT: Biomimetic platforms for human stem cell research. Cell Stem Cell 2011, 8:252-261.
5. Yim EK, Sheetz MP: Force-dependent cell signaling in stem cell differentiation. Stem Cell Res Ther 2012, 3:41.

6. Manivannan S, Nelson CM: Dynamics of branched tissue assembly. Stem Cell Res Ther 2012, 3:42.

7. Conway A, Schaffer DV: Biophysical regulation of stem cell behavior within the niche. Stem Cell Res Ther 2012, 3:50.

8. Fridley KM, Kinney MA, MCDevitt TC: Hydrodynamic modulation of pluripotent stem cells. Stem Cell Res Ther 2012, 3:45.

9. Wan LQ, Ronaldson K, Guirguis M, Vunjak-Novakovic G: Micropatterning of cells reveals chiral morphogenesis. Stem Cell Res Ther 2013, 4:24.

10. Serbo JV, Gerecht S: Vascular tissue engineering: biodegradable scaffold platforms to promote angiogenesis. Stem Cell Res Ther 2013, 4:8.

11. Hung BP, Hutton DL, Grayson WL: Mechanical control of tissueengineered bone. Stem Cell Res Ther 2013, 4:10.

12. Thavandiran N, Nunes SS, Xiao Y, Radisic M: Topological and electrical control of cardiac differentiation and assembly. Stem Cell Res Ther 2013, 4:14.

13. de Peppo GM, Marolt D: Modulating the biochemical and biophysical culture environment to enhance osteogenic differentiation and maturation of human pluripotent stem cell-derived mesenchymal progenitors. Stem Cell Res Ther 2013, 4:106.

14. Chen JC, Jacobs CR: Mechanically induced osteogenic lineage commitment of stem cells. Stem Cell Res Ther 2013, 4:107.

15. O'Conor CJ, Case N, Guilak F: Mechanical regulation of chondrogenesis. Stem Cell Res Ther 2013, 4:61.

16. Schiller ZA, Schiele NR, Sims JK, Lee K, Kuo CK: Adipogenesis of adipose-derived stem cells may be regulated via the cytoskeleton at physiological oxygen levels in vitro. Stem Cell Res Ther 2013, 4:79.

17. Zimmermann WH: Biomechanical regulation of in vitro cardiogenesis for tissue-engineered heart repair. Stem Cell Res Ther 2013, 4:137.

\section{$10.1186 /$ scrt383}

Cite this article as: Vunjak-Novakovic: Physical influences on stem cells. Stem Cell Research \& Therapy 2013, 4:153 